\title{
Monoclonal gammopathy associated with multiple myeloma and visceral leishmaniasis in the dog: A comparison of two cases
}

\author{
M. T. Antognoni • F. Birettoni • A. Miglio • P. Lalli • \\ F. Porciello $\cdot$ V. Mangili Pecci
}

Published online: 13 May 2010

(C) Springer Science+Business Media B.V. 2010

\begin{abstract}
The term monoclonal gammopathy (MG) suggests the presence of clonal immunoglobulins in blood serum that are recognized as narrow spikes in the $\beta$ and/or $\gamma$ region of the electrophoretic pattern of serum. In the dog, MG is rare and is associated with a heterogeneous group of diseases that include multiple myeloma (the most common source of $\mathrm{MG}$ ) as well as infectious and chronic inflammatory diseases such as Leishmaniasis. In this paper, two cases of MG are described: the first case is associated with multiple myeloma of monoclonal component type $\operatorname{IgA} / \lambda$, with the latter rare in $\operatorname{dogs}$, and the second case involves MG that developed 3 years after an initial diagnosis of Leishmaniasis.
\end{abstract}

Keywords Dog $\cdot$ Monoclonal gammopathy $\cdot$ Multiple myeloma $\cdot$ Visceral leishmaniasis

\begin{abstract}
Abbreviations
MG Monoclonal gammopathy

MC Monoclonal component
\end{abstract}

\section{Introduction}

The term monoclonal gammopathy (MG) is suggestive of the presence of clonal immunoglobulins in the serum, which can be recognized as a narrow spike in the $\beta$ and/ or $\gamma$ regions in the electrophoretic blood pattern. These paraproteins are produced by a single B lymphocyte clone, which yields an excessive amount of immunoglobulins or their subunits, as kappa $(\kappa)$ or lambda $(\lambda)$ heavy and light chains. The identification of a MG in the dog is rare and is usually associated with multiple myeloma (main cause of $\mathrm{MG}$ ), Waldenström macroglobulinemia, chronic lymphocytic leukemia, plamocytic leukemia, amyloidosis, chronic pyodermitis, ehrlichiosis, and leishmaniasis (Giraudel et al. 2002; Matus et al. 1986). In our clinical practice, the identification of MG allowed us to refine the

M. T. Antognoni $(\varangle) \cdot$ F. Birettoni $\cdot$ A. Miglio $\cdot$ F. Porciello $\cdot$ V. Mangili Pecci

Department of Pathology, Diagnostic and Veterinary Clinic, University of Perugia, Perugia, Italy e-mail: mariateresa.antognoni@unipg.it

P. Lalli

Perugia Medical Hospital, Perugia, Italy 
diagnostic procedure in two dogs, one affected by multiple myeloma (MM) with monoclonal component (MC) type $\operatorname{Ig} \mathrm{A} / \lambda$, and the other affected by visceral leishmaniasis (L) in which the MG was detected 3 years after disease onset.

\section{Materials and methods}

\section{Animals}

Case $N^{\circ} 1$ A 9-year-old, male, mixed-breed dog had been showing depression, panting, disorexia, polyuria, polydipsia, and exophthalmos of the right eye for 2 weeks. Direct clinical examination revealed a poor state of nutrition and pale mucous membranes combined with suffusion, ulceration, and bleeding on the labial mucosa, splenomegaly, and hypertensive uveitis of the right eye.

Case $N^{\circ} 2$ A 7-year-old, female, mixed-breed dog was affected by L since November 2005. This dog received N-metilglutamina antimoniate $(100 \mathrm{mg} / \mathrm{kg}$ SID intramuscularly for 1 month, initiated three times, with 40-50 days between each course), and allopurinol (15 mg/ kg BID orally for 7 months), and benazepril $(0.25 \mathrm{mg} / \mathrm{kg}$ SID orally), which was sufficient to cause remission of symptoms and normalization of blood counts and clinical biochemistry within 1 year. However, serological positivity against L (indirect immunofluorescence: IFI 1:320) persisted. The animal presented a first relapse of disease in January 2008 , for which she underwent miltefosine administration ( $2 \mathrm{mg} / \mathrm{kg}$ orally for 28 days), and a second relapse 1 year later, requiring further observation.

\section{Laboratory and instrumental examinations}

Blood samples were used to obtain blood counts (Genius Vet SEAC), clinical biochemistry values (Hitachi 704 and Roche kits), serum and urine protein electrophoresis results [sodium dodecyl sulphate acrylamide gel electrophoresis (SDS-AGE)-Hydrasys liquid chromatography (LC), Sebia and Hydragel $\beta 1-\beta 2$ kits], and serum immunofixation (IFS) electrophoresis results (SDS-AGE-Hydrasys LC, Sebia and human Hydragel IF kits) using antisera to anti-heavy chains (gamma-IgG, alpha-IgA, and mu-IgM) and to anti- $k$ and $-\lambda$ light chains (free and bound). Blood serum samples were used to determine antibody titers against Leishmania infantum, Ehrlichia canis, Anaplasma phagocytophilum, and Rickettsia rickettsii by IFI, while whole blood samples were processed for the detection of A. platys by polymerase chain reaction (PCR). Urine samples obtained by cystocentesis were used for physical and chemical examination and for the cytological assessment of sedimentation rates after centrifugation at $1,500 \mathrm{rpm}$ for $5 \mathrm{~min}$ and the urinary protein to urinary creatinine ratio (PU/CU) [SDS-AGE, Hydragel proteinuria and high-resolution (HR) kits]. Urinary immunofixation (IFU) electrophoresis [SDS-AGE-Hydrasys LC, Sebia and Hydragel Bence Jones (BJ) kits] was performed using antisera against trivalent anti heavy chain gamma (IgG), alpha (IgA), and mu (IgM), anti-k free and bound light chains, and anti- $\lambda$ free and bound light chains. Samples from fine needle aspiration of lymph nodes and bone marrow were used for cytological evaluation, after preparation of smears stained with May Grünwald Giemsa. Ultrasound examinations of the abdomen and skeletal X-rays were performed in both dogs. Systolic blood pressure was detected using the Doppler technique, and evaluation of serum viscosity was detected only in Case $\mathrm{N}^{\circ} 1$. 


\section{Results}

Both dogs showed normocytic, normochromic anemia (RBC 4.6-5.76 M/ $\mu \mathrm{L}, \mathrm{Hb} 9.1-$ $12.9 \mathrm{~g} / \mathrm{dL}$, and HCT 26.5-36.5\%), thrombocytopenia (36-158 K/uL), leukopenia (5.5$5.8 \mathrm{~K} / \mu \mathrm{L})$, hyperproteinemia (14.2-14.5 g\%), and hypoalbuminemia (12.4-18.0\%). Serum protein electrophoresis results showed a $\mathrm{MC}$ in the $\beta 2-\gamma(71.2 \%)$ globulin fraction in Case $\mathrm{N}^{\circ} 1$ (Fig. 1) and in the $\gamma$ globulin fraction (71.4\%) in Case $\mathrm{N}^{\circ} 2$ (Fig. 2). IFS results showed a $\mathrm{MC}$ as an $\operatorname{Ig} \mathrm{A} / \lambda$ type in Case $\mathrm{N}^{\circ} 1$ and an $\mathrm{IgG} / \lambda$ type in Case $\mathrm{N}^{\circ} 2$. Quantification of MCs by densitometric scanning of the areas under the peak evaluated using HR urinary electrophoresis was $72.4 \mathrm{~g} / \mathrm{L}$ in Case $\mathrm{N}^{\circ} 1$ and $91.6 \mathrm{~g} / \mathrm{L}$ in Case $\mathrm{N}^{\circ} 2$. In both dogs, there was mixed, glomerular and tubular, incomplete proteinuria $(189.6$ and $178 \mathrm{mg} / \mathrm{dL}$, respectively), and PU/CU values were pathological (4.51 and 2.78 , respectively). IFU results revealed the same MCs in urine and BJ proteinuria of $\lambda$ type in Case $\mathrm{N}^{\circ} 2$ (HR quantification of BJ proteinuria was $384.8 \mathrm{mg} / \mathrm{L}$ ). Bone marrow cytology showed severe plasmocytosis in case $\mathrm{N}^{\circ} 1$ (35\% atypical plasma cells, including anisocytosis, anisokaryosis, and multinucleation) and moderate plasmocytosis (15\%) associated with evidence of Leishmania organisms in Case $\mathrm{N}^{\circ} 2$. PCR was negative for $\mathrm{L}$ in contrast to serological IFI that was positive against $\mathrm{L}$ in Case $\mathrm{N}^{\circ} 2$. Radiographic images revealed no osteolytic bone lesions. Serum viscosity and systolic blood pressure in Case $\mathrm{N}^{\circ} 1$ were $8.2 \mathrm{sec}$ and $220 \mathrm{~mm}$ $\mathrm{Hg}$, respectively. Therapy instituted in Case $\mathrm{N}^{\circ} 1$ included the use of benazepril $(0.5 \mathrm{mg} / \mathrm{kg}$ SID orally) and prednisone (1 $\mathrm{mg} / \mathrm{kg}$ SID for 7 days orally, then $0.5 \mathrm{mg} / \mathrm{kg}$ SID) for 4 months, allowing only partial and transient remission of symptoms; therefore, melphalan $(0.1 \mathrm{mg} / \mathrm{kg}$ SID orally for 10 days then $0.05 \mathrm{mg} / \mathrm{kg}$ SID) was added. Two months after initiation of melphalan treatment, serum and urine abnormalities resolved, and there were no MCs on serum protein electrophoresis, even though its presence was still detectable with IFS ( $3 \mathrm{~g} / \mathrm{L})$ and IFU. On bone marrow cytology, plasmocytosis was still detectable $(25 \%$ atypical plasma cells) in association with the clinical signs observed at the first observation.

\section{Discussion}

IFS and IFU are considered the gold standards in both human and veterinary medicine for the detection of MCs. In our case, the commercial kit developed for human use revealed the presence of MCs and BJ proteinuria, showing cross-reactivity between antisera against

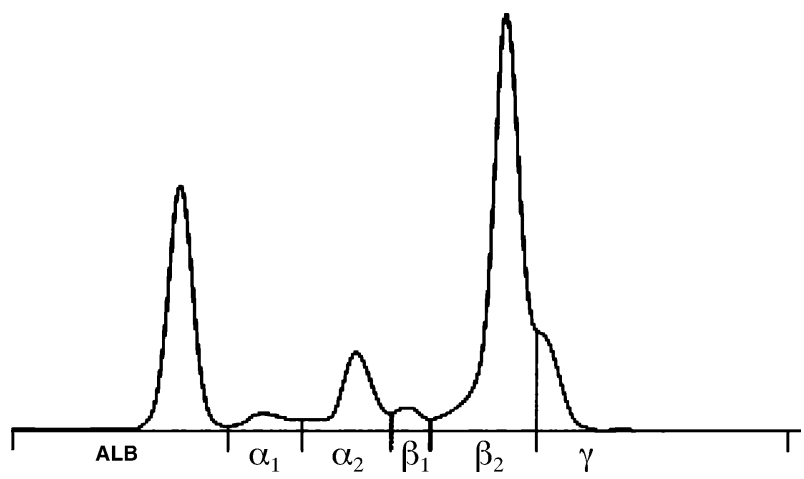

Fig. 1 Case $\mathrm{N}^{\circ} 1$. Evidence of a monoclonal component in the $\beta 2-\gamma$ globulin fraction 


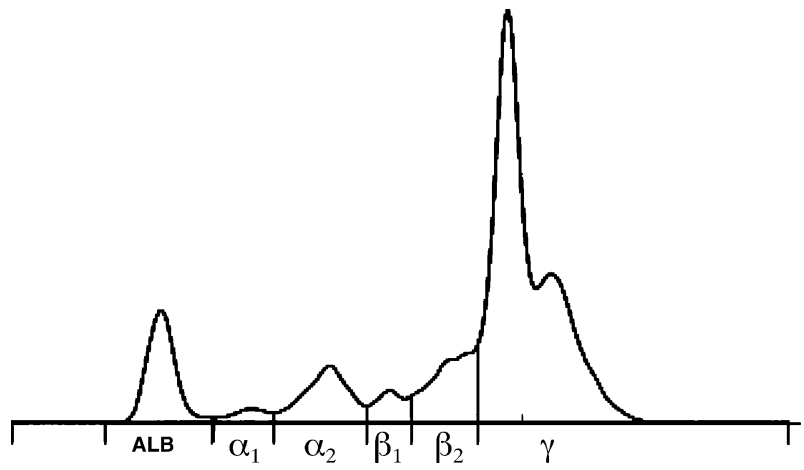

Fig. 2 Case $\mathrm{N}^{\circ} 2$. Evidence of a monoclonal component in the $\gamma$ globulin fraction

human immunoglobulins and canine immunoglobulins. In the first case, the presence of an $\operatorname{IgA} / \lambda$ type $\mathrm{MC}$ in serum and urine added to the characteristic bone marrow findings, allowing us to diagnosis MM, stage I (Matus et al. 1986). The rate of MM occurrence is less than $1 \%$ of all canine tumors, $8 \%$ of all hematopoietic neoplasms, and the IgA type occurs in 50\% of cases (Cayzer and Jones 1991; Cowgill et al. 2004; Hendrix et al. 1998; Matus et al. 1986; Ramaiah et al. 2002). MM can be diagnosed based on the presence of at least two of the four following criteria: paraproteinemia, bone marrow plasmocytosis over 5-20\%, osteolytic bone lesions, and BJ proteinuria. In the first case, the absence of osteolytic bone lesions is in agreement with data found in the literature (Mayer et al. 2008; Matus et al. 1986) in which these injuries have been documented in 50\% of dogs (Cayzer and Jones, 1991; Hendrix et al. 1998). Serum hyperviscosity and its associated syndrome (i.e., hypertension, eye damage, kidney disease, and bleeding) are attributable to the presence of an IgA type MC (Hendrix et al. 1998; Matus et al. 1986). The remission of symptoms after melphalan administration is in agreement with data reported by Cayzer and Jones (1991), Hendrix et al. (1998), and Matus et al. (1986). Cayzer and Jones (1991) also reported that the average survival time of patients treated with prednisone alone or in combination with melphalan is 220 and 540 days, respectively, and that there is a significant correlation between survival time and response to therapy. It has also been reported that $90 \%$ of subjects treated with prednisone combined with melphalan respond positively and that survival time is not influenced by either continuous or intermittent (during periods of burning or disease relapse) treatment (Cayzer and Jones 1991; Hendrix et al. 1998; Mayer et al. 2008 ). Osteolytic lesions, hypercalcemia, and BJ proteinuria are considered negative prognostic factors in course of MM (Cowgill et al. 2004; Hendrix et al. 1998; Ramaiah et al. 2002) and are effectively absent in Case $\mathrm{N}^{\circ} 1$. There are few reports, in either human or veterinary medicine, about the occurrence of $\mathrm{L}$ in the presence of a $\mathrm{MC}$, so that $\mathrm{L}$ is rarely taken into consideration within a differential diagnosis (Bonfanti et al. 2004; Font et al. 1994). In human medicine (Randi et al. 2006; Rombolà et al. 2008), a few cases of an early diagnosis of MM have been reconsidered based on both the detection of $\mathrm{L}$ amastigoti in the bone marrow and remission of symptoms after antiparasitic therapy. The appearance of MG in dog $\mathrm{N}^{\circ} 2$ after treatment and approximately 3 years after diagnosis is similar to a case of $\mathrm{L}$ in a man described by Rombolà et al. (2008), in which the paraproteinemia appeared after therapy had been terminated for 6 months. Rombolà et al. (2008), Bonfanti et al. (2004), and Giraudel et al. (2002) explain this correlation as the result of a chronic antigenic stimulation in a genetically susceptible patient. 
The identification of an IgG/ $\lambda$ type $\mathrm{MC}$ in the dog infected with $\mathrm{L}$ is in agreement with the rare cases reported in the literature (Font et al. 1994; Giraudel et al. 2002; Randi et al. 2006). Particularly important is also the detection of BJ proteinuria of $\lambda$ type by IFU. Furthermore, the lack of correspondence between the position of a BJ-related band and those of the heavy and light chains of the MC identified in serum is consistent with the activity of a single plasma cell clone producing only light immunoglobulin chains. This finding is reported in human medicine during micromolecular myeloma, a condition characterized by the presence of only BJ proteinuria in the absence of MG (Bonfanti et al. 2004; Font et al. 1994). For these reasons, the dog affected by L is rechecked periodically. In conclusion, we highlight that the finding of a MG, as emphasized in human medicine, should lead to specific medical investigations that are relevant for differential diagnosis, prognosis, and therapy. In addition, in patients with $\mathrm{MG}$, it is important to quantify MC serum concentrations and $\mathrm{BJ}$ proteinuria to follow disease progression and monitor tumor burden and efficacy of treatment using the same methods (Hendrix et al. 1998).

\section{References}

Bonfanti U, Zini E, Minetti E, Zatelli A (2004) Free light-chain proteinuria and normal renal histopatology and function in $11 \mathrm{dogs}$ exposed to Leishmania infantum, Ehrlichia canis, and Babesia canis. J Vet Intern Med 18:618-624

Cayzer J, Jones BR (1991) IgA multiple myeloma in a dog. N Z Vet J 39:139-144

Cowgill ES, Neel JA, Ruslander D (2004) Light-chain myeloma in a dog. J Vet Intern Med 18:119-121

Font A, Closa JM, Mascort J (1994) Monoclonal gammopathy in a dog with visceral leishmaniasis. J Vet Intern Med 8:233-235

Giraudel JM, Pages JP, Guelfi JF (2002) Monoclonal gammopathies in the dog: A retrospective study of 18 cases (1986-1999) and literature review. J Am Anim Hosp Assoc 38:135-147

Hendrix DVH, Gelatt KN, Smith PJ, Brooks DE, Whittaker CJG, Chmielewski NT (1998) Ophthalmic disease as the presenting complaint in five dogs with multiple myeloma. J Am Anim Hosp Assoc 34:121-128

Matus RE, Leifer CE, MacEwen EG, Hurvitz AI (1986) Prognostic factors for multiple myeloma in the dog. J Am Vet Med Assoc 11:1288-1291

Mayer MN, Kerr ME, Grier CK, MacDonald VS (2008) Immunobulin A multiple myeloma with cutaneous involvement in a dog. Can Vet J 49:694-702

Ramaiah SK, Seguin MA, Carwile HF, Raskin RE (2002) Biclonal gammopathy associated with immunoglobulin A in a dog with multiple myeloma. Vet Clin Pathol 31:83-89

Randi ML, Ruzzon E, Tezza F, Pacquola E, Fabris F (2006) Monoclonal gammopathy in human leishmaniasis. Neth J Med 64:50-51

Rombolà F, Spinoso A, Bertuccio SN (2008) Monoclonal gammopathy after visceral leishmaniasis: just a coincidence? Infez Med 3:173-174 